\title{
Motion Control of a Wheeled Millirobot
}

\author{
Rachel Drisdelle, Zendai Kashino, Laura Pineros, Justin Y. Kim, Goldie Nejat, Beno Benhabib \\ Department of Mechanical and Industrial Engineering, University of Toronto \\ 5 King's College Road, Toronto, Ontario, M5S 3G8 Canada \\ zendkash@mie.utoronto.ca
}

\begin{abstract}
This paper presents a new closed-loop control system for a novel wheeled millirobot, mROBerTO, developed at the University of Toronto. The proposed system was verified via vision-based robot-pose tracking, a centralized computer for directing movement, and a firmware PID controller. Further modification to the original robot structure, via the addition of stabilizing arms, was made to improve tracking performance. Numerous motion-control experiments were performed to evaluate the performance and robustness of the controller on the millirobot. The system presented is simple, requires little knowledge of robot's motion characteristics, and consumes limited processing capabilities. Furthermore, experimental results show that the system can provide accurate motion control for various trajectories despite the limitations that result from the small footprint of mROBerTO, $16 \times 16 \times 32$ $\mathrm{mm}^{3}$.
\end{abstract}

Keywords: Millirobots, closed-loop control, PID control, locomotion.

\section{Introduction}

Millirobots can have a range of applications, including wireless sensor networks (WSNs) [1], micro-assembly [2], medicine [3], urban search and rescue [4], wilderness search and rescue [5], [6], [7] and surveillance [8]. Centralized control of these small-scale robots, when possible, can lead to improved coordination between individual robots [9]. However, the control of millirobot motion can be challenging due to, typically, nonlinear behaviour, high likelihood of misalignment during manufacturing, limited self-sensing capabilities and computational power.

Two basic types of controllers have been proposed for robot motion control [10]: kinematics based [11] versus dynamics based [12]. Dynamic controllers tend to outperform kinematic controllers, but, require real-time information regarding the robot's state and behaviour [13]. This can make them unadaptable to millirobots where knowledge about the robot state is often limited and characterizing robot dynamics can be difficult.

In this paper, we present a new centralized, closed-loop motion control system designed for use with the mROBerTO millirobot developed in our lab, [14]. Additionally, we present a novel modification to mROBerTO to improve motion control performance. The milli-robot-Toronto (mROBerTO) is unique among other wheeled millirobots due to its relatively small size and method of locomotion. Centralized control of the robot is achieved by performing trajectory tracking through posture stabilization, specifying the trajectory as a set of goal configurations. Closed-loop feedback of robot position and orientation is achievable through an external positioning system, while motion control is handled by a firmware PID controller. The control method presented herein can be applied to any millirobot for centralized control.

\section{Robot Overview}

The millirobot, mROBerTO, developed in our laboratory, Fig. 1, has a footprint of $16 \times 16 \times 32 \mathrm{~mm}^{3}$, and was designed with the objective of achieving increased modularity to allow future improvement and expansion; maximum use of commercially available components for ease of production, assembly and maintenance; and, minimum possible footprint area without sacrificing processing power and sensing capabilities, [14].

\subsection{Hardware and Design}

mROBerTO comprises four modules: mainboard, primary sensing, secondary sensing, and locomotion. The mainboard is the central processing and communication module that connects to all other modules and handles primary wireless communication to external devices. It utilizes the Nordic nRF51422 system-on-chip (SoC) - a 32-bit ARM 
Cortex-M0, with built-in Bluetooth ${ }^{\circledR}$ Smart and ANT $+{ }^{\mathrm{TM}}$ capability.

The primary sensing module includes a CMOS camera and a time-of-flight proximity sensor. The Toshiba TCM8230MD camera can take videos in VGA resolution at $30 \mathrm{fps}$ and outputs RGB565 or YUV422 data via eight parallel data pins. The VL6180X is a 3-in-1, proximity sensing, ambient light sensing, and laser source unit. The secondary sensing module, located on top of the robot, includes two LEDs, a gyroscope, and a magnetometer.

Two 4-mm Nano coreless motors are connected to an H-bridge for a differential drive configuration. The shafts of the motors are directly in contact with the floor surface and act as 'wheels'. A $3.175 \mathrm{~mm}$ diameter polytetrafluoroethylene ball is used as the rear third contact point with the floor. The heaviest components, three $3.7 \mathrm{~V} \mathrm{Li-Po} \mathrm{batteries,} \mathrm{are} \mathrm{placed}$ vertically above the motors.

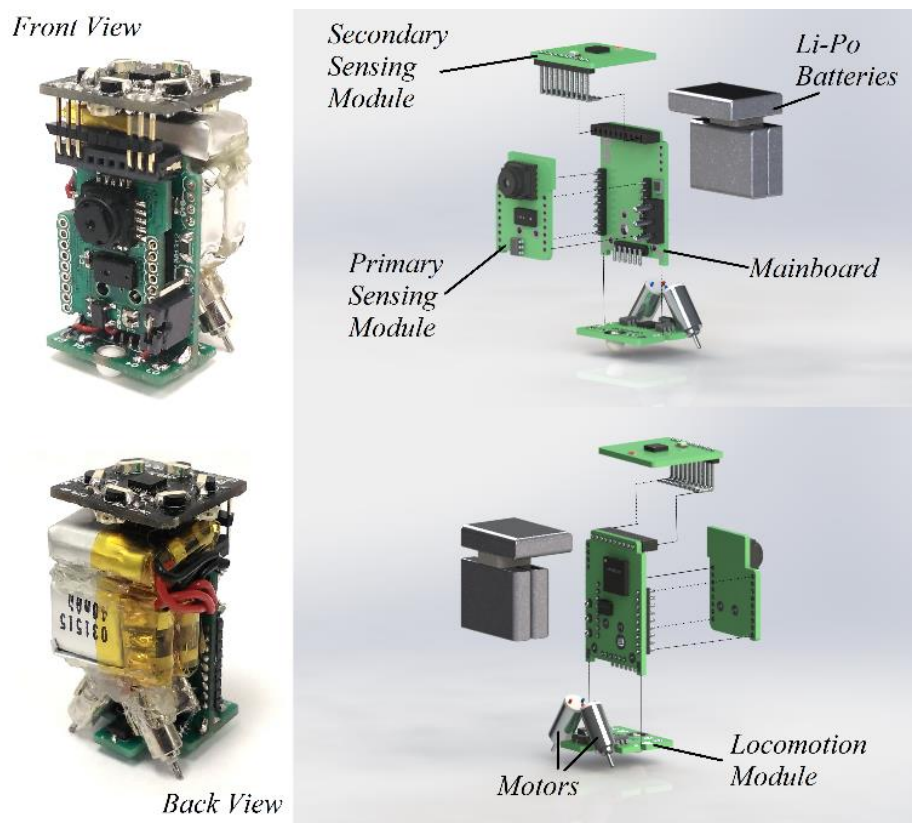

Fig. 1: mROBerTO exploded assembly.

\subsection{Open-Loop Motion Characteristics}

In order to, first, evaluate the open-loop motion characteristics of the robot, motion with constant PWM output to both motors was observed. This evaluation was performed with various PWM levels, starting positions and orientations to determine factors that may affect robot motion. Fig. 2 shows a representative set of three open-loop motion trajectories in which the robot started at the same position and orientation (pose) and was directed to output a 20\% duty cycle PWM to each motor.

When required to move on a line, the uncalibrated robot veered to the left as shown in Fig. 2(a), while in Fig. 2(b) and (c), it veered to the right. Reasons for this non-linear behaviour include motor misalignments during assembly, surface conditions, and differences in motor characteristics.

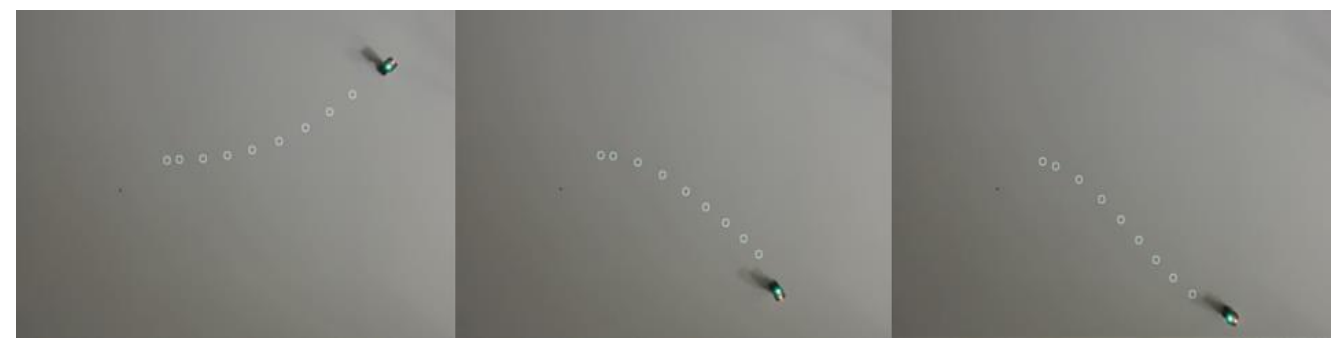

(a)

(b)

(c)

Fig. 2: Three open-loop linear motion trajectories. 


\section{Closed-Loop Control System}

In order to demonstrate the potential closed-loop control behaviour of the millirobot, we needed a method of determining robot pose in the workspace as well as a controller to direct robot motion. Since mROBerTO, like most other millirobots, lacks the ability to independently perform odometry, an external positioning system is required to provide it with feedback regarding motion. In our system, a vision-based system was used to determine robot pose in the workspace. The information obtained was relayed to the robot through a central computer along with the robot's desired position every $0.75 \mathrm{~s}$. In addition to this $1.3 \mathrm{~Hz}$ visual feedback, information from the on-board gyroscope was used to estimate the current robot orientation at $100 \mathrm{~Hz}$. A controller implemented in the robot's firmware, then, directed robot motion based on the robot's current pose and its desired pose.

The controller implemented in the robot firmware is a PID motion controller with decoupled pose error:

$$
\boldsymbol{u}(t)=\boldsymbol{K}_{\boldsymbol{p}} \boldsymbol{e}(t)+\boldsymbol{K}_{\boldsymbol{i}} \int_{0}^{t} \boldsymbol{e}(\tau) d \tau+\boldsymbol{K}_{\boldsymbol{d}} \frac{d \boldsymbol{e}(t)}{d t}
$$

where

$$
\boldsymbol{u}(t)=\left[\begin{array}{c}
\dot{x} \\
\dot{y} \\
\dot{\phi}
\end{array}\right], \boldsymbol{e}(t)=\left[\begin{array}{l}
x_{g}-x \\
y_{g}-y \\
\phi_{g}-\phi
\end{array}\right], \boldsymbol{K}_{\boldsymbol{p}}=\left[\begin{array}{c}
K_{p x} \\
K_{p y} \\
K_{p \phi}
\end{array}\right], \boldsymbol{K}_{\boldsymbol{i}}=\left[\begin{array}{c}
K_{i x} \\
K_{i y} \\
K_{i \phi}
\end{array}\right], \boldsymbol{K}_{\boldsymbol{d}}=\left[\begin{array}{l}
K_{d x} \\
K_{d y} \\
K_{d \phi}
\end{array}\right] \text {. }
$$

Herein, $(x, y)$ and $\phi$ refer to the robot's current position and orientation, respectively, $\left(x_{g}, y_{g}\right)$ and $\phi_{g}$ refer to the desired (goal) position and orientation, respectively, all in a global coordinate frame. $K_{p x}, K_{i x}, K_{d x}, K_{p y}, K_{i y}, K_{d y}, K_{p \phi}, K_{i \phi}$, and $K_{d \phi}$ refer to the proportional, integral, and derivative gain constants for position and orientation, respectively. $\phi_{g}$ is calculated as the direction in which the robot should move to reach the next goal position:

$$
\phi_{g}=\operatorname{atan} 2\left(y_{g}-y, x_{g}-x\right)
$$

A differential drive kinematic model [15] was used to translate the output of the PID controller into wheel angular velocities:

$$
\omega_{l}=(2 v-\dot{\phi} L) / 2 R, \omega_{r}=(2 v+\dot{\phi} L) / 2 R,
$$

where $L$ is the length of the axel, $R$ is the radius of the wheels, and $v$ is defined as:

$$
v=\sqrt{\dot{x}^{2}+\dot{y}^{2}}
$$

An upper limit was placed on $\omega_{l}$ and $\omega_{r}$ to improve stability [16]. In order to convert angular velocity $\omega$ to PWM percentage values, the nonlinear relation between the effective wheel angular velocity and PWM was modelled through an empirical relationship obtained from studying the open-loop motion characteristics of the robot. The PWM percentage required to achieve a given angular velocity $\omega$ for the left and right wheels $\left(P W M_{L}\right.$ and $\left.P W M_{R}\right)$ were found to be approximately given by:

$$
P W M_{L}\left(\omega_{l}\right)=4.2 \log \left(\omega_{l}+0.3\right)+5, P W M_{R}\left(\omega_{r}\right)=4.5 \log \left(\omega_{r}+0.4\right)+4.1 .
$$

\section{Stabilizing Arms}

As the millirobot traverses through trajectories with small turning radii, the instantaneous angular velocities of the millirobot, $w_{o}$, could be relatively high. Due to the non-linear behaviour arising from slight irregularities in the millirobot, these instances may result in irregular responses from the controller. The addition of stabilizing arms, one on each side of the millirobot, Fig. 3, showed to improve this behaviour by acting as a damper. The addition of these arms, the mass of which is less than $1 \%$ of the millirobot, increases the moment of inertia, which, due to a conservation of angular momentum, decreases the angular velocity, $w_{f}[17]$. 


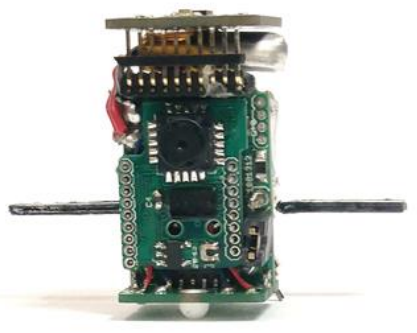

Fig. 3: mROBerTO fitted with stabilizing arms.

The geometrical model of the millirobot, without the addition of the stabilizing arms, can be simplified so that the moment of inertia is described by:

$$
L=\left(I_{r}+m_{r} d_{r}^{2}\right) w_{o}
$$

where $I_{r}$ is the robot's moment of inertia, $m_{r}$ is the mass of the robot, $d_{r}$ is the distance from the robot's centre vertical axis to the axis of rotation.

The inclusion of the stabilizing arms changes the moment of inertia to be:

$$
L=\left(I_{r}+m_{r} d_{r}^{2}+2\left(I_{d}+m_{d} d_{d}^{2}\right)\right) w_{f},
$$

where $I_{d}$ is the stabilizing arm's moment of inertia, $m_{d}$ is the mass of each arm, and $d_{d}$ is the distance from the axis of rotation to the centre vertical axis of the damper.

In order to conserve momentum, we equate Eq. (7) to Eq. (8), which yields:

$$
\frac{w_{f}}{w_{o}}=\left(I_{r}+m_{r} d_{r}^{2}\right) /\left(I_{r}+m_{r} d_{r}^{2}+2\left(I_{d}+m_{d} d_{d}^{2}\right)\right) \text {. }
$$

Using the dimensional properties of the robot and the stabilizing arms, where each arm is $15 \times 1 \times 1 \mathrm{~mm}^{3}$, made of polyethylene with a mass of $0.071 \mathrm{~g}$, the decrease in the millirobot's angular velocity is less than $2 \%$.

\section{Motion Performance}

Numerous experiments were performed to evaluate closed-loop motion control performance. In each experiment, the robot was directed to follow a different trajectory. Since robot motion is directed by sending goal poses to the robot every $0.75 \mathrm{~s}$, each trajectory needs to be discretized. Fig. 4 shows two representative examples of actual robot paths taken (red) superimposed on the desired path (black).

\section{Conclusions}

This paper presents a simple closed-loop control system for the centralized control of the mROBerTO millirobot. The control system utilizes a vision-based robot tracking system, a centralized computer to provide feedback to and control the robot, and a PID controller implemented on the millirobot firmware. The paper also presents a novel modification to the mROBerTO millirobot that improves motion control performance. Despite the low rate of feedback regarding robot position and nonlinear, inconsistent open-loop motion characteristics, the control system and novel modification allows the robot to follow both $(a)$ smooth and $(b)$ sharp-cornered trajectories. 


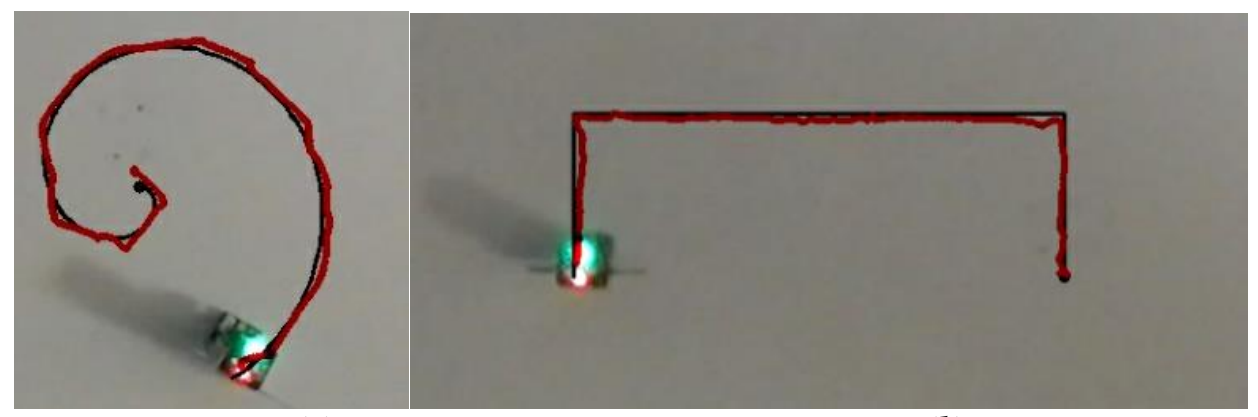

(a)

(b)

Fig. 4: Actual robot paths.

\section{Acknowledgements}

This research was funded in part by the Natural Sciences and Engineering Research Council of Canada, and the Canada Research Chairs Program.

\section{References}

[1] K. Lembke, Ł. Kietliński, M. Golański, and R. Schoeneich, "RoboMote: Mobile autonomous hardware platform for Wireless Ad-hoc Sensor Networks," in Proceedings of the IEEE International Symposium on Industrial Electronics, Gdansk, Poland, pp. 940-944, 2011.

[2] P. Vartholomeos, K. Vlachos, and E. Papadopoulos, "Analysis and motion control of a centrifugal-force microrobotic platform," IEEE Transactions on Automation Science and Engineering, vol. 10, no. 3, pp. 545-553, 2013.

[3] A. W. Mahoney and J. J. Abbott, "Five-degree-of-freedom manipulation of an untethered magnetic device in fluid using a single permanent magnet with application in stomach capsule endoscopy," The International Journal of Robotics Research, vol. 35, no. 1-3, pp. 129-147, 2016.

[4] R. S. Fearing, "Challenges for effective millirobots," in Proceedings of the IEEE International Symposium on MicroNanoMechanical and Human Science, Nagoya, Japan, pp. 1-5, 2006.

[5] Z. Kashino, J. Y. Kim, G. Nejat, and B. Benhabib, "Spatiotemporal adaptive optimization of a static-sensor network via a non-parametric estimation of target location likelihood," IEEE Sensors Journal, vol. 17, no. 5, pp. 1479-1492, 2017.

[6] Z. Kashino, J. Vilela, J. Y. Kim, G. Nejat, and B. Benhabib, "An adaptive static-sensor network deployment strategy for detecting mobile targets," in Proceedings of the IEEE International Symposium on Safety, Security, and Rescue Robotics, Lausanne, Switzerland, pp. 1-8, 2016.

[7] A. Macwan, J. Vilela, G. Nejat, and B. Benhabib, "Multi-robot deployment for wilderness search and rescue," International Journal of Robotics and Automation, vol. 31, no. 1, pp. 45-51, 2016.

[8] S. Bergbreiter, "Effective and efficient locomotion for millimeter-sized microrobots," in Proceedings of the IEEE/RSJ International Conference on Intelligent Robots and Systems, Nice, France, pp. 4030-4035, 2008.

[9] A. Macwan, J. Vilela, G. Nejat, and B. Benhabib, "A multirobot path-planning strategy for autonomous wilderness search and rescue," IEEE Transactions on Cybernetics, vol. 45, no. 9, pp. 1784-1797, 2014.

[10] C. Y. Sosa-Cervantes, R. Silva-Ortigoza, C. Márquez-Sánchez, H. Taud, and G. Saldaña-González, "Trajectory tracking task in wheeled mobile robots: A review," in Proceedings of the International Conference on Mechatronics, Electronics and Automotive Engineering, Cuernavaca, Mexico, pp. 110-115, 2014.

[11] D.-H. Kim and J.-H. Oh, "Tracking control of a two-wheeled mobile robot using input-output linearization," Control Engineering Practice, vol. 7, no. 3, pp. 369-373, 1999.

[12] S. G. Tzafestas, "3 - Mobile robot dynamics," in Introduction to Mobile Robot Control, Oxford: Elsevier, pp. 69-99, 2014.

[13] A. Bessas, A. Benalia, and F. Boudjema, "Integral sliding mode control for trajectory tracking of wheeled mobile robot in presence of uncertainties," Journal of Control Science and Engineering, 2016, doi: http://dx.doi.org/10.1155/2016/7915375. 
[14] J. Y. Kim, T. Colaco, Z. Kashino, G. Nejat, and B. Benhabib, "mROBerTO: A modular millirobot for swarmbehavior studies," in Proceedings of the IEEE/RSJ International Conference on Intelligent Robots and Systems, Daejeon, Korea, pp. 2109-2114, 2016.

[15] R. Velázquez and A. Lay-Ekuakille, "A review of models and structures for wheeled mobile robots: Four case studies," in Proceedings of the International Conference on Advanced Robotics, Tallinn, Estonia, pp. 524-529, 2011.

[16] Y. Kanayama, Y. Kimura, F. Miyazaki, and T. Noguchi, "A stable tracking control method for an autonomous mobile robot," in Proceedings of the IEEE International Conference on Robotics and Automation Proceedings, Cincinnati, OH, vol. 1, pp. 384-389, 1990.

[17] B. Denardo, "Demonstration of the parallel-axis theorem," The Physics Teacher, vol. 36, no. 1, p. 56, 1998. 